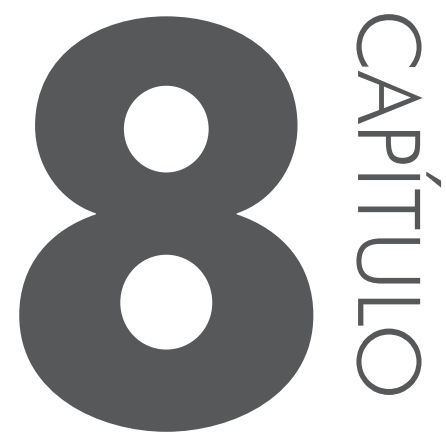

\title{
MODELAGEM CINEMÁTICA, SIMULAÇÃO DE MOVIMENTO, CONSTRUÇÃO E CONTROLE DE PRÓTESE DE MÃO PARA
} TAREFAS DE MANIPULAC̣ÃO

\author{
Marcelo H. Stoppa \\ Universidade Federal de Goiás, \\ Instituto de Matemática e Tecnologia. \\ mhstoppa@pq.cnpq.br
}

João Carlos Mendes Carvalho

Universidade Federal de Uberlândia, Faculdade de Engenharia Mecânica. jcmendes@mecanica.ufu.br

\section{RESUMO}

Vários modelos matemáticos têm sido utilizados para a representação da mão humana. Eles descrevem modelos com até 26 graus de liberdade (GDL), os quais são necessários para obter uma representação eficiente para tarefas de manipulação realizada por próteses de mão. Contudo, um grande número de GDL resulta em um sistema complexo e, em geral, necessita de simplificação. Consequentemente, modelos simplificados envolvem erros quando é necessário replicar as posturas da mão em tarefas diárias. Este artigo analisa um modelo proposto pelo projeto InMoov (open source), cuja mão robótica possui cinco dedos, capaz de produzir um arco palmar, com um total de $17 \mathrm{GDL}$. Aqui é descrito o modelo cinemático completo para essa mão artificial, para o qual as equações do modelo são encontradas por meio das coordenadas de Denavit-Hartenberg. O modelo executa apenas movimento de flexão das juntas, e os dedos polegar, indicador e 
médio têm 3 links e três articulações de rotação que são responsáveis pela flexão. Os dedos anelar e mínimo possuem 4 links e 4 articulações de rotação, o que permite uma condição de arco palmar. Em seguida, são realizadas simulações computacionais para comparar o modelo real ao modelo matemático na realização de alguns movimentos de preensão da mão. Para finalizar, são exibidos os procedimentos construtivos da mão avaliada, cujos componentes estruturais são manufaturados em impressora 3D, seguidos do detalhamento do sistema eletrônico de controle.

Palavras-chaves: Cinemática, Prótese de mão, Modelagem, InMoov, Impressão 3D.

\section{INTRODUÇÃO}

Apesar de não existirem dados precisos no Brasil, Shibata e Mendes (2012) afirmam que cerca de 50 mil pessoas sofreram algum tipo de amputação pelo Sistema Único de Saúde (SUS) em 2011. Destes, cerca de 33\% foram decorrentes de causas externas, ou seja, acidentes.

A amputação é um termo utilizado para definir a retirada total ou parcial de um membro, a qual, além de ser diagnóstico em casos de acidentes, pode ser um método de tratamento para diversas doenças. De acordo com Carvalho (2003) e O’Sullivan e Schmitz (2004), as amputações por traumas são mais comuns em acidentes de trânsito e em ferimentos por armas de fogo.

As pessoas amputadas, principalmente de membros superiores, geralmente se deparam com uma grande dificuldade de readaptação pós-trauma, uma vez que se tornam incapazes de executar tarefas antes muito simples. Essa incapacidade acaba por influenciar negativamente sua vida e sua interação social. Escovar os dentes, passar o fio dental, amarrar os sapatos, fechar o cinto, dar um aperto de mão e muitas outras atividades do dia a dia passam a ser obstáculos que acabam por deprimir os novos amputados, dificultando muito a sua recuperação e reinserção social.

De acordo com Kulley (2003), muitas dessas pessoas poderiam se beneficiar psicologicamente, melhorando sua autoestima, simplesmente vislumbrando uma maior possibilidade física por meio da utilização de uma prótese. É muito triste o fato de que essas pessoas ainda são vistas como "diferentes" na sociedade, promovendo um sentimento de compaixão, chegando a ser colocadas num patamar de inutilidade. Entretanto, essas pessoas simplesmente querem se socializar sendo tratadas normalmente, mostrando que são capazes de levar uma vida normal e que ainda são úteis. Amputados são pessoas fortes e competentes, que podem fazer muito com o que têm e que são capazes de superar a adversidade.

Ainda há espaço para melhoria em todos aspectos da atual tecnologia de próteses, tanto em matéria de concepção mecânica como em processamento de 
dados (sinais elétricos), bem como em relação ao desempenho geral do sistema. Segundo Ventimiglia (2012), não há muitas grandes empresas de desenvolvimento de próteses porque o mercado ainda é muito pequeno e limitado em relação a perspectivas de negócio.

Por outro lado, comercialmente, encontram-se tanto próteses muito simples do tipo gancho ou com finalidade puramente estética quanto próteses robóticas de alto custo e pouco utilizadas.

Dellon e Matsuoka (2007) afirmam que num futuro próximo, a necessidade por dispositivos robóticos de assistência deve aumentar, tendo em vista que a população mundial acima de 65 anos, hoje $20 \%$ do total, tende a chegar a $35 \%$ em 2015 (enquanto na década de 1950 esse percentual era de apenas 4,9\%). Esse "envelhecimento" geral da população deve impor um cuidado maior com os riscos de saúde oriundos do aumento da idade biológica. De maneira geral, as soluções robóticas se apresentam como uma opção na ajuda da resolução desses problemas, permitindo que o idoso recupere sua qualidade de vida e adquira uma maior independência. Outro fator de peso é a afirmação de Shibata e Mendes (2012) de que um alto percentual de amputações são consequências de sintomas da idade, como diabetes e problemas vasculares (tromboses).

A utilização de próteses para suprir um membro amputado é muito antiga, fato comprovado pela existência de uma múmia, da época dos antigos egípcios, dotada de um dedão do pé feito de madeira e acoplado ao pé por meio de placas de madeira e fitas de couro. Observou-se um aumento no interesse por membros artificiais durante a Guerra Civil Americana, provavelmente devido ao grande número de amputações ocorridas naquele evento. Um fato que proporcionou um melhor ajuste das próteses foi a descoberta dos anestésicos, que passaram a permitir um tempo maior de cirurgia, proporcionando maior possibilidade de ajuste protético aos médicos.

Com o passar do tempo, os modelos de próteses tornaram-se mais leves e mais adaptáveis, incluindo novos materiais e sistemas eletromecânicos. Atualmente as próteses evoluíram tanto que pessoas usuárias de membros inferiores têm até participado de esportes de alto-desempenho. Contudo, a complexidade do movimento das mãos tem colocado as próteses de membros superiores, em especial as próteses de mão, num patamar de necessidade tecnológica que ainda torna inviável a popularização de próteses de maior funcionalidade.

Quando se trata de pesquisa em próteses de mão, busca-se um projeto de prótese que responda aos anseios fisiológicos de velocidade e força, capaz de ser controlado à resposta do pensamento. Contudo isto está muito longe de ser alcançado, e a prótese passa a ser uma ferramenta e não um substituto ao membro perdido.

Os fatores mais limitantes para as próteses, na prática, são o peso, a força e as restrições dos sistemas de controle necessários aos graus de liberdade do sis- 
tema mecânico. De acordo com Weir (2004), as dificuldades do projeto de uma mão artificial são muito mais desafiadoras do que as de um robô industrial, por exemplo. Ele afirma que o projeto de próteses e a robótica têm muito menos em comum do que se poderia esperar e os conceitos de robótica tiveram pouco impacto sobre próteses comerciais por causa das limitações físicas significativamente diferentes necessárias para que uma prótese pudesse ser bem-sucedida.

É notório que o projeto de mãos artificiais demande esforços multidisciplinares, já que necessita do entendimento da mecânica de mecanismos, tais como engrenagens e alavancas, além de acionamentos eletromecânicos, switches, motores e eletrônicos. Nesse contexto, a mecatrônica é a palavra de ordem. Em adição à mecatrônica, o projeto de próteses de mão carece de conhecimentos acerca de anatomia musculoesquelética e de neurofisiologia.

No que concerne às pesquisas de desenvolvimento de próteses de membro superior, Harvey e Longstaff (2001) desenvolveram a base para uma prótese de mão que opera como uma parte da rede neural corporal, com o diferencial de proporcionar ao usuário um feeedback tátil, em que a transformação dos sinais de entrada/saída é efetuada por meio de um sistema referencial de coordenadas. Eles propuseram uma simplificação a apenas 6 graus de liberdade, mantendo, contudo, os movimentos mais importantes da mão.

A respeito da interação homem-prótese, Peleg, Braiman, Yom-Tov e Inbar (2002) usaram sinais eletromiográficos gravados por dois pares de eletrodos colocados na superfície do braço para controle de prótese, de que vários recursos foram extraídos de modo a classificar a ativação dos dedos da prótese. As características mais relevantes foram selecionadas para serem usadas como entradas de algoritmo genético, resultando numa probabilidade de erro inferior a $2 \%$.

Posteriormente, Shenoy, Miller, Crawford e Rao (2008) investigaram o uso de sinais eletromiográficos superficiais para controle em tempo real de um braço robótico com uma precisão entre 92 e $98 \%$.

De modo geral, nesse ambiente de próteses de alta funcionalidade, o cenário comum ou é o de pesquisas em centros acadêmicos de desenvolvimento, cujos resultados acabam por ficar longe de aplicações efetivamente populares, ou, por outro lado, em grandes corporações, cujos produtos são lançados comercialmente a valores elevados que se colocam passíveis de aquisição por uma ínfima parcela da população. Em resumo, as próteses de alta funcionalidade, que sem dúvida viriam agregar uma melhor qualidade de vida a amputados, não passam de sonho, chegando a se posicionar num patamar de ficção científica, muito distante da realidade.

Diante do exposto, este projeto apresenta o estudo e o desenvolvimento de próteses de mão eletromecânicas, compostas de cinco dedos funcionais, incluindo 
rotação de polegar, com vistas à readaptação de pessoas amputadas cujo cotovelo é preservado, levando-se em conta o projeto de um protótipo funcional de baixo custo que possa atender pessoas de menor poder aquisitivo.

Diversos estudos têm sido realizados no que concerne à construção de partes do corpo humano que possam substituir, artificialmente, órgão lesionados ou amputados.

Nesse contexto, Belter et al. (2013) apresentam um review que exibe uma análise detalhada das características mecânicas de próteses antropomórficas de mão. Eles reportam um estudo empírico sobre o desempenho de várias próteses de mão comercialmente disponíveis, tais como Vincent, iLimb, iLimb Pulse, Bebionic, Bebionic v2 e Michelangelo. São discutidos e comparados vários parâmetros de projeto, incluindo número de atuadores, complexidade, peso e força de preensão.

Como base para os estudos de movimentos da mão, Cutkosky e Wright (1986) apresentam uma classificação para as tarefas de manipulação, examinando as pegadas usadas por humanos quando estes trabalham com ferramentas e partes metálicas. As pegadas são comparadas em termos força, área de contato, atrito, amortecimento e sensibilidade tátil. A comparação permitiu a criação de uma taxonomia de pegadas, que é a base para o início dos estudos de movimentos de mão.

No que diz respeito à cinemática de próteses de mão, Cobos et al. (2008) propõem um modelo cinemático com 25 GDL, baseado no esqueleto humano. As restrições dinâmicas e estáticas foram incluídas de modo a melhorar o realismo dos movimentos. Eles ainda apresentam dois modelos simplificados com número de GDL reduzidos e com restrições predefinidas, mas que apresentam erros na repetição da postura desejada. Esses erros são calculados com respeito ao modelo de 24 GDL e avaliados de acordo com os gestos avaliados.

Ainda na análise cinemática, Grebenstein et al. (2010), também tomando como base um esqueleto humano, incorporam restrições estruturais e variações incrementais, de modo a melhorar os parâmetros funcionais necessários para a construção de uma prótese de mão. Os testes foram derivados de um conjunto de pegadas comuns, algumas das quais utilizadas por cirurgiões no processo pós-operatório de mãos. Eles constroem alguns protótipos de papel-cartão para avaliação dos resultados cinemáticos, que são posteriormente utilizados no DLR Hand Arm System.

Posteriormente, Cobos et al. (2010) publicam um capítulo de livro, em que apresentam um estudo completo da cinemática direta e inversa de mão, avaliando as relações entre os dedos. São avaliadas, ainda, as relações entre diversas simplificações de modelos contemplando os erros relativos entre estes. 
Na sequência deste trabalho, é descrito o modelo cinemático completo para uma mão artificial, proposto pelo projeto InMoov, em que as equações do modelo são encontradas por meio das coordenadas de Denavit-Hartenberg. Essa prótese permite o movimento de flexão das juntas, e os dedos polegar, indicador e médio têm 3 links e três articulações de rotação que são responsáveis pela flexão. Os dedos anelar e mínimo possuem 4 links e 4 articulações de rotação, o que permite uma condição de arco palmar.

\section{FORMULAC̣ÃO DO MODELO}

Como base para este projeto, optou-se por analisar e avaliar possibilidades de utilização de protótipos existentes que pudessem, contudo, guardar a característica primordial da proposta, que é o baixo custo.

O projeto InMoov, que tem a característica Open Source, proposto pelo modelista francês Gael Langevin, envida esforços em torno da produção de um robô humanoide em tamanho real e disponibilizado para download pela internet, o projeto de todas as partes que podem ser impressas em uma impressora 3D. Portanto, em oposição aos altos custos de uma prótese comercial, e objetivando a construção de uma prótese mais barata, como ponto de partida, buscando um projeto viável, foram utilizadas as partes referentes à mão robótica, disponibilizadas pelo InMoov (LANGEVIN, 2014).

A primeira etapa do estudo foi a análise cinemática com posterior simulação computacional do projeto de mão robótica InMoov, para posterior adaptação e uso como prótese de mão. Na sequência, é descrita a análise cinemática do modelo.

O modelo de prótese de mão analisado aqui é baseado no esqueleto humano, com algumas simplificações. Enquanto a mão humana tem mais de $20 \mathrm{GDL}$, o modelo avaliado é composto por 12 links que simulam os correspondentes ossos humanos, e 17 GDL representam as articulações de rotação que, para simplificação, doravante serão chamadas apenas de juntas. Aqui, é mantida a notação e nomenclatura em inglês por questão de conveniência, de modo a não alterar as figuras, equações e tabelas, em função dos artigos que foram escritos e publicados em língua inglesa. A notação utilizada para o modelo considera $1_{\mathrm{ij}}$ e $\theta_{\mathrm{ij}}$, respectivamente, como links e juntas, onde i representa um dedo e j representa uma junta ou link, de acordo com o que mostra a Tabela 1 .

Portanto, assumindo essa configuração, os dedos, polegar (thumb) T, indicador (index) I, e médio (middle) $\mathrm{M}$ têm três links e três juntas cada um, enquanto os dedos anelar (ring) R e mínimo (little) L têm quatro juntas e quatro links cada, num total de 17 GDL. Os GDL $\theta_{\mathrm{L}, \mathrm{CMC}}$ e $\theta_{\mathrm{R}, \mathrm{CMC}}$ permitem simular o arco da palma da mão, que é um tipo de deformação que acontece quando a prótese pega uma bola ou objetos similares a este. 


\subsection{Convenção de Denavit-Hartenberg}

Quando é considerado um número reduzido de GDL em um projeto de prótese de mão, é necessário melhorar o projeto de modo que o manuseio de objetos, nas suas mais variadas formas, possa ser realizado de modo efetivo. Assim, o projeto mecânico é um aspecto importante, que carece de atenção especial e a simulação pode ser de grande ajuda nesse sentido. A modelagem matemática é o primeiro passo para estabelecer uma boa simulação.

Tabela 1 Notação para juntas e links no modelo

\section{DEDOS}

\begin{tabular}{cccccc}
\hline Juntas & Mínimo (L) & Anelar (R) & Médio (M) & Indicador (I) & Polegar (T) \\
\hline $\begin{array}{c}\text { interfalangeal distal (DIP) } \\
\text { interfalangeal proximal }\end{array}$ & $\theta_{L, D I P}$ & $\theta_{R, D I P}$ & $\theta_{M, D I P}$ & $\theta_{I, D I P}$ & - \\
(PIP) & $\theta_{L, P I P}$ & $\theta_{R, P I P}$ & $\theta_{M, P I P}$ & $\theta_{I, P I P}$ & - \\
metacarpofalangeal (MCP) & $\theta_{L, M C P}$ & $\theta_{R, M C P}$ & $\theta_{M, M C P}$ & $\theta_{I, M C P}$ & $\theta_{T, M C P}$ \\
carpometacarpal (CMC) & $\theta_{L, C M C}$ & $\theta_{R, C M C}$ & - & - & - \\
interfalangeal (IP) & - & - & - & - & $\theta_{T, I P}$ \\
trapeziometacarpal (TMC) & - & - & - & - & $\theta_{T, T M C}$ \\
\hline Links & Mínimo (L) & Anelar (R) & Médio (M) & Indicador (I) & Polegar (T) \\
\hline metacarpal (MC) & $\ell_{L, M C}$ & $\ell_{R, M C}$ & - & - & $\ell_{T, M C}$ \\
proximal (P) & $\ell_{L, P}$ & $\ell_{R, P}$ & $\ell_{M, P}$ & $\ell_{I, P}$ & $\ell_{T, P}$ \\
medial (M) & $\ell_{T, M}$ & $\ell_{R, M}$ & $\ell_{M, M}$ & $\ell_{I, M}$ & - \\
distal (D) & $\ell_{T, D}$ & $\ell_{R, D}$ & $\ell_{M, D}$ & $\ell_{I, D}$ & $\ell_{T, D}$ \\
\hline
\end{tabular}

Na sequência, é descrita a modelagem matemática acerca dos aspectos cinemáticos da prótese, que é o estudo do movimento sem levar em conta as causas do movimento (TARZIMI et al., 2009). As equações do modelo são calculadas por meio da convenção modificada de Denavit-Hartenberg (KHALIL; KLEINFINGER, 1986), em que os sistemas de referência recebem rótulos de acordo com a junta sobre a qual estão posicionados. Nesse procedimento, o eixo $Z_{\mathrm{i}}$ está sobre o eixo da junta $i$, denominado por $\mathrm{L}_{\mathrm{i}}$ e o corpo $\mathrm{C}_{\mathrm{i}}$ está associado a um referencial $i$. 
A metodologia consiste em:

1) Identificar os eixos $\mathrm{L}_{i}$ de todas as juntas.

2) $\mathrm{O}_{\mathrm{i}}$ é o pé da perpendicular comum aos eixos das juntas $\mathrm{L}_{\mathrm{i}}$ e $\mathrm{L}_{\mathrm{i}+1}$, situado sobre $\mathrm{L}_{\mathrm{i}}$. Se os eixos das duas articulações são paralelos ou coincidentes, pode-se escolher arbitrariamente uma perpendicular comum. Nesse caso, considerações de simetria e/ou de simplicidade permitem uma escolha adequada.

3) $X_{i}$ é um vetor unitário dessa perpendicular comum, orientado de $L_{i}$ na direção da articulação $\mathrm{L}_{\mathrm{i}+1}$. Se os eixos dessas duas articulações são concorrentes ou coincidentes, a orientação é arbitrária. Entretanto, considerações de simetria e/ou de simplicidade permitem uma escolha adequada.

4) $Z_{i}$ é um vetor unitário do eixo $L_{i}$, orientado arbitrariamente.

5) $\mathrm{Y}_{\mathrm{i}}$ é definido pelo produto vetorial positivo.

Aqui, são considerados apenas movimentos de flexão e 3 diferentes configurações cinemáticas:

I. Para o polegar, com 3 juntas e 3 links, na junta trapeziometacarpal existe um sistema de referência cujos eixos não são mutuamente paralelos às juntas metacarpofalangeal e interfalangeal (Figura 1).

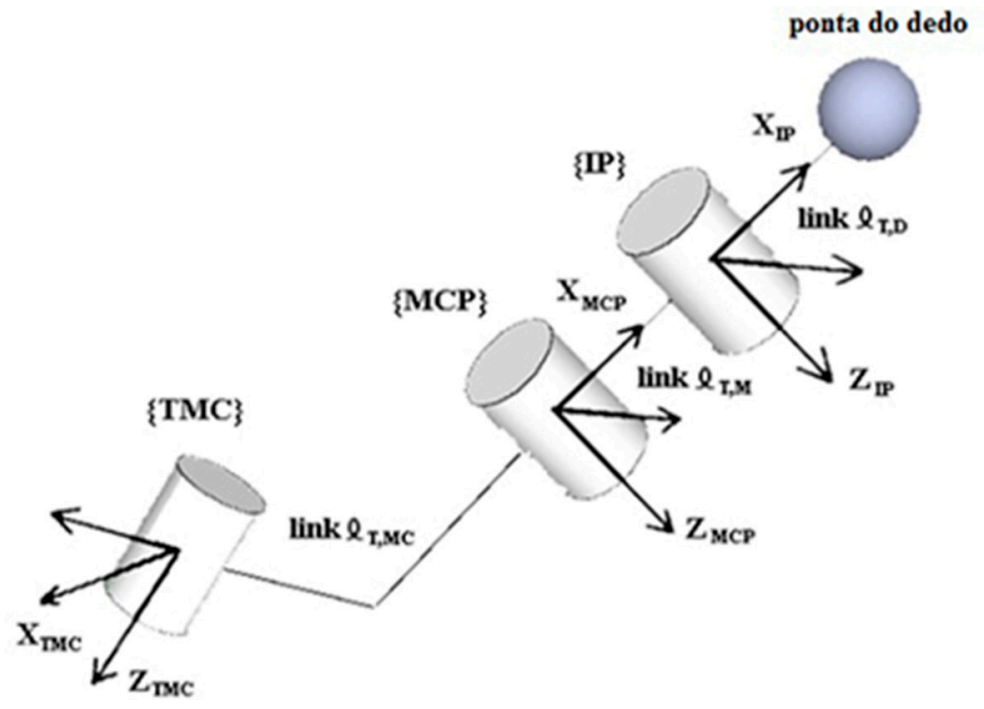

Figura 1 Modelo para o polegar.

II. Para os dedos indicador e médio (i = I, M), também com 3 juntas e 3 links, todos os sistemas de referência tem eixos mutuamente paralelos (Figura 2).

II. Para os dedos anelar e mínimo ( $\mathrm{i}=\mathrm{R}, \mathrm{L}$ ), com 4 juntas e 4 links, o sistema carpometacarpal (CMC) tem eixos não mutuamente paralelos aos outros sistemas (Figura 3). 


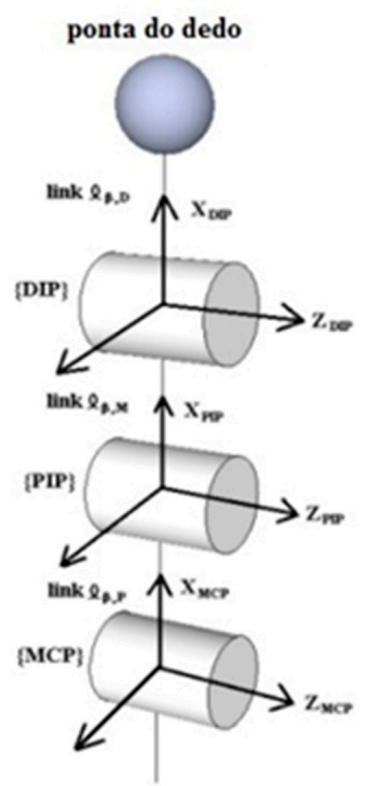

Figura 2 Modelo para os dedos indicador e médio.

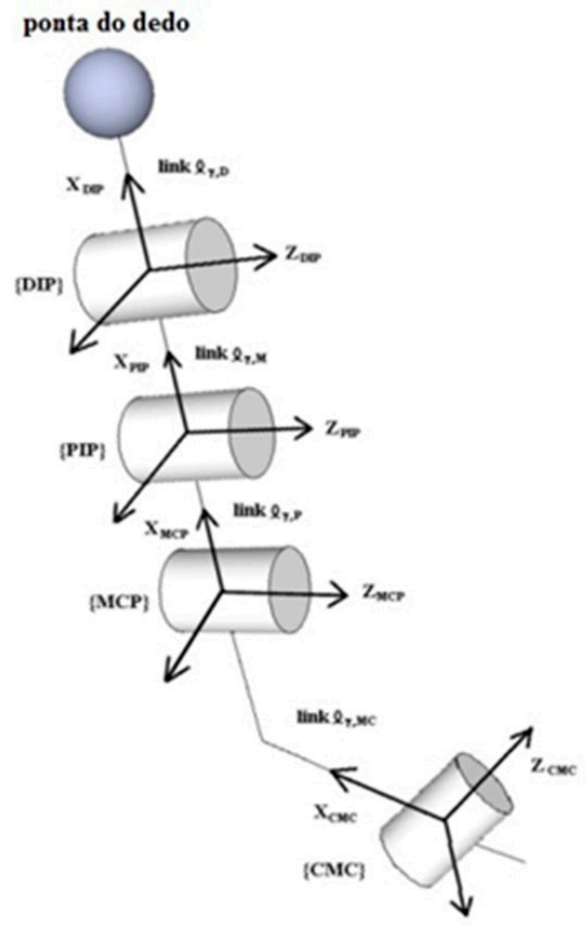

Figura 3 Modelo para os dedos anelar e mínimo. 
A Figura 4 exibe o modelo completo com os 5 dedos, 17 GDL e em que um referencial inercial é posicionado no centro do punho.

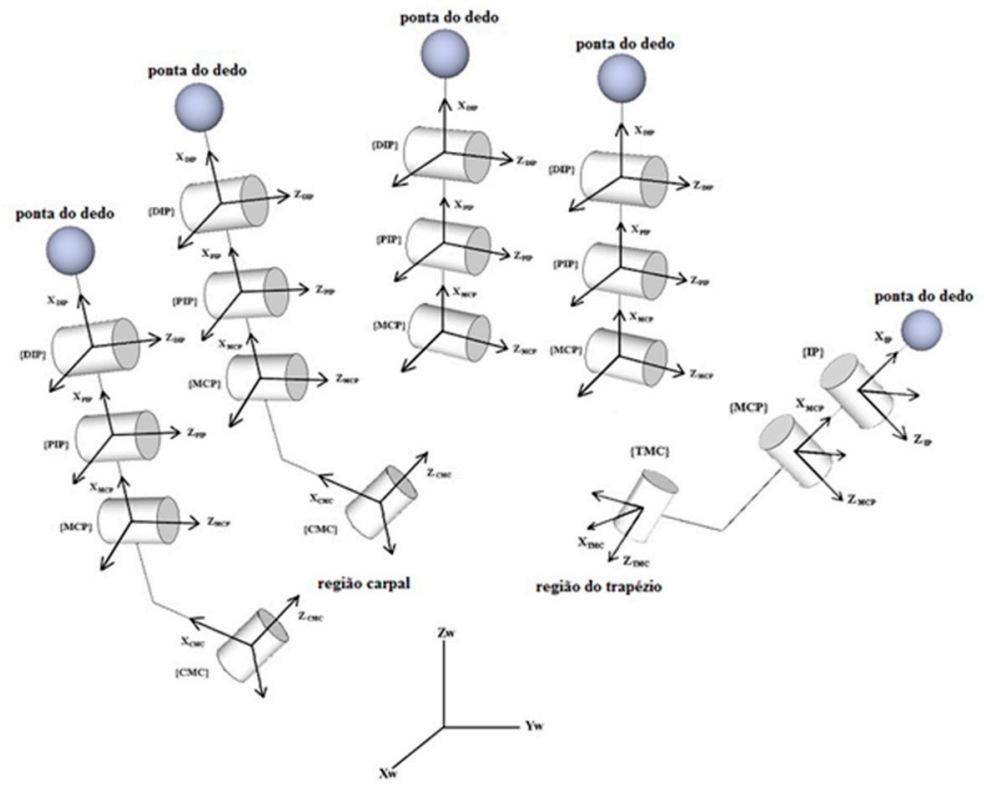

Figura 4 Cinemática completa da prótese considerada.

\subsection{Cinemática direta}

A solução para a cinemática direta de cada dedo é encontrada por meio de matrizes homogêneas. Elas são usadas para determinar a posição e a orientação das pontas dos dedos relativas ao sistema de coordenadas inercial, localizado no centro do punho. As equações do modelo são calculadas por meio dos parâmetros de Denavit-Hartenberg (D-H), em que a terminologia anatômica humana é usada para descrever o modelo da prótese. Considerando as 3 configurações, como na Seção 2.1, os parâmetros D-H são determinados de acordo com a convenção proposta por Khalil e Kleinfinger (1986). As variáveis do modelo $\left(\theta_{\mathrm{ij}}\right)$ representam o ângulo de flexão em cada junta. Em todos os parâmetros D-H, i e j são índices que representam, respectivamente, o dedo e a junta correspondente. Após alocar os sistemas de coordenadas de acordo com a convenção D- $\mathrm{H}$, os parâmetros $\theta_{\mathrm{i}}$, $\mathrm{d}_{\mathrm{i}}, \alpha_{\mathrm{i}}$ e $\mathrm{r}_{\mathrm{i}}$ são tais que:

- $\theta_{\mathrm{i}}$ é o ângulo entre $X_{\mathrm{i}-1}$ e $X_{\mathrm{i}}$ em torno do eixo $Z_{\mathrm{i}}$;

- $\mathrm{d}_{\mathrm{i}}$ é a distância entre os eixos $Z_{\mathrm{i}-1}$ e $Z_{\mathrm{i}}$

- $\alpha_{\mathrm{i}}$ é o ângulo entre $Z_{\mathrm{i}-1}$ e $Z_{\mathrm{i}}$ em torno do eixo $X_{\mathrm{i}-1}$;

- $r_{i}$ é a distância entre $X_{i-1}$ e $X_{i}$, ao longo de $Z_{i}$. 
Para calcular a posição da ponta do dedo, é necessário supor um novo referencial ali alocado, considerando apenas uma translação do referencial anterior. Os ângulos $\theta_{\mathrm{i}}$ e $\alpha_{\mathrm{i}}$ são definidos positivos aplicando-se a regra da mão direita.

\subsubsection{Cinemática do polegar}

A cinemática para o polegar é definida de acordo com os parâmetros da Tabela 2, como mostrado na Equação (1).

$$
P T={ }_{0}^{\omega} T_{T}\left(r_{T}, \omega\right){ }_{1}^{0} T_{T}\left(\theta_{T}, T M C\right){ }_{2}^{1} T_{T}\left(\theta_{T},,_{M C P}\right){ }_{3}^{2} T_{T}\left(\theta_{T},{ }_{I P}\right){ }_{4}^{2} T_{T}\left(\theta_{T},{ }_{D}\right)
$$

onde $\mathrm{P}_{\mathrm{T}}$ representa a matriz que contém a posição e orientação da ponta do polegar com respeito ao centro do punho; $\mathrm{T}_{\mathrm{T}}$ são as matrizes que representam as contribuições de translação ou rotação de cada junta, dada, em um forma geral (CRAIG, 2004), por:

$$
{ }_{k}^{k-1} T_{T}\left(\theta_{i, j}\right)=\left[\begin{array}{cccc}
\cos \left(\theta_{i, j}\right) & -\sin \left(\theta_{i, j}\right) & 0 & d_{i, j} \\
\cos \left(\alpha_{i, j}\right) \sin \left(\theta_{i, j}\right) \cos \left(\alpha_{i, j}\right) \cos \left(\theta_{i, j}\right)-\sin \left(\alpha_{i, j}\right)-r_{i, j} \sin \left(\alpha_{i, j}\right) \\
\sin \left(\alpha_{i, j}\right) \sin \left(\theta_{i, j}\right) \sin \left(\alpha_{i, j}\right) \cos \left(\theta_{i, j}\right)-\cos \left(\alpha_{i, j}\right)-r_{i, j} \cos \left(\alpha_{i, j}\right) \\
0 & 0 & 0 & 1
\end{array}\right]
$$

Tabela 2 Parâmetros D-H para o polegar

\begin{tabular}{ccccc}
\hline iunta & $\theta_{\mathrm{i}, \mathrm{i}}$ & $\mathrm{d}_{\mathrm{i}, \mathrm{i}}$ & $\mathrm{r}_{\mathrm{i}, \mathrm{i}}$ & $\alpha_{\mathrm{i}, \mathrm{i}}$ \\
\hline $\mathrm{W}$ & 0 & 0 & $\mathrm{r}_{\mathrm{T}, \mathrm{W}}$ & 0 \\
1 & $\theta_{\mathrm{T}, \mathrm{MCC}}$ & 0 & $\mathrm{r}_{\mathrm{T}, M C}$ & $\alpha_{\mathrm{T}, \mathrm{MC}}$ \\
2 & $\theta_{\mathrm{T}, M C P}$ & 0 & $\mathrm{r}_{\mathrm{T}, \mathrm{P}}$ & $\alpha_{\mathrm{T}, \mathrm{P}}$ \\
3 & $\theta_{\mathrm{T}, \mathrm{P}}$ & $\mathrm{d}_{\mathrm{T}, \mathrm{P}}$ & 0 & 0 \\
4 & 0 & $\mathrm{~d}_{\mathrm{T}, \mathrm{D}}$ & 0 & 0 \\
\hline
\end{tabular}

Então, cada uma das matrizes $\mathrm{T}_{\mathrm{T}}$ são dadas por:

$$
{ }_{0}^{\omega} T_{T}\left(r_{T},{ }_{\omega}\right)=\left[\begin{array}{cccc}
1 & 0 & 0 & 0 \\
0 & 1 & 0 & 0 \\
0 & 0 & 1 & r_{T}, \omega \\
0 & 0 & 0 & 1
\end{array}\right]
$$




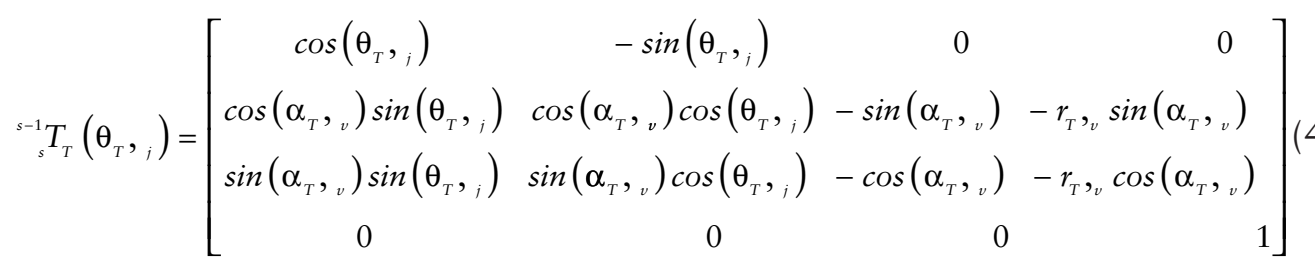

onde $(s=1,2),(v=M C, P)$ e $(j=$ TMC, MCP), nessa ordem.

$$
\begin{gathered}
{ }_{3}^{2} T_{T}\left(\theta_{T},{ }_{I P}\right)=\left[\begin{array}{cccc}
\cos \left(\theta_{T},{ }_{I P}\right) & -\sin \left(\theta_{T},{ }_{I P}\right) & 0 & d_{T},{ }_{P} \\
\sin \left(\theta_{T},{ }_{I P}\right) & \cos \left(\theta_{T},{ }_{I P}\right) & 0 & 0 \\
0 & 0 & 1 & 0 \\
0 & 0 & 0 & 1
\end{array}\right] \\
{ }_{4}^{3} T_{T}\left(\theta_{T, D}\right)=\left[\begin{array}{cccc}
0 & 0 & 0 & d_{T, D} \\
0 & 1 & 0 & 0 \\
0 & 0 & 1 & 0 \\
0 & 0 & 0 & 1
\end{array}\right]
\end{gathered}
$$

\subsubsection{Cinemática dos dedos indicador e médio}

Analogamente à Seção 2.2.1, a cinemática direta para os dedos indicador e médio são definidas de acordo com os parâmetros da Tabela 3, como mostrado na Equação (7), onde ( $\beta=\mathrm{I}, \mathrm{M})$ (Index, Middle).

Tabela 3 Parâmetros D-H para os dedos indicador e médio

$$
\begin{aligned}
& \begin{array}{ccccc}
\hline \text { iunta } & \theta_{\mathrm{i}, \mathrm{i}} & \mathrm{d}_{\mathrm{i}, \mathrm{j}} & \mathrm{r}_{\mathrm{i}, \mathrm{j}} & \alpha_{\mathrm{i}, \mathrm{j}} \\
\hline \mathrm{W} & 0 & 0 & \mathrm{r}_{\mathrm{b}, \mathrm{w}} & 0 \\
1 & \theta_{\beta, M C P} & 0 & \mathrm{r}_{\mathrm{b}, M C} & \alpha_{\mathrm{b}, M C} \\
2 & \theta_{\beta, P P} & \mathrm{~d}_{\beta, P} & 0 & 0 \\
3 & \theta_{\beta, D P} & \mathrm{~d}_{\beta, M} & 0 & 0 \\
4 & 0 & \mathrm{~d}_{\beta, D} & 0 & 0 \\
\hline
\end{array} \\
& P_{\beta}={ }_{0}^{\omega} T_{\beta}\left(r_{\beta, \omega}\right){ }_{1}^{0} T_{\beta}\left(\theta_{\beta, M C P}\right){ }_{2}^{1} T_{\beta}\left(\theta_{\beta, P I P}\right){ }_{3}^{2} T_{\beta}\left(\theta_{\beta, D I P}\right){ }_{4}^{3} T_{\beta}\left(\theta_{\beta, D}\right)
\end{aligned}
$$

onde $\mathrm{P}_{\beta}$ representam as matrizes que contém a posição e orientação das pontas dos dedos indicador e médio, com respeito ao centro do punho; $\mathrm{T}_{\beta}$ são as matrizes como aquelas da Equação (2), aqui dadas por: 


$$
\begin{gathered}
{ }_{1}^{0} T_{\beta}\left(\theta_{\beta, M C P}\right)=\left[\begin{array}{cccc}
-\sin \left(\theta_{\beta, M C P}\right) & -\sin \left(\theta_{r},{ }_{i}\right) & 0 & 0 \\
\cos \left(\alpha_{\beta, M C P}\right) \sin \left(\theta_{\beta, M C P}\right) & \cos \left(\alpha_{\beta, M C P}\right) \cos \left(\theta_{\beta, M C P}\right) & -\sin \left(\alpha_{\beta, M C P}\right) & -r_{\beta, M C P} \sin \left(\alpha_{\beta, M C P}\right) \\
\sin \left(\theta_{\beta, M C P}\right) \sin \left(\theta_{\beta, M C P}\right) & \sin \left(\alpha_{\beta, M C P}\right) \cos \left(\alpha_{\beta, M C P}\right) & \cos \left(\alpha_{\beta, M C P}\right) & r_{\beta, M C P} \cos \left(\alpha_{\beta, M C P}\right) \\
0 & 0 & 0 & 1
\end{array}\right] \\
{ }_{s}^{s} T_{\beta}\left(\theta_{\beta, j}\right)=\left[\begin{array}{cccc}
\cos \left(\theta_{\beta, j}\right) & -\sin \left(\theta_{\beta, j}\right) & 0 & d_{\beta, \nu} \\
\sin \left(\theta_{\beta, j}\right) & \cos \left(\theta_{\beta, j}\right) & 0 & 0 \\
0 & 0 & 1 & 0 \\
0 & 0 & 0 & 1
\end{array}\right]
\end{gathered}
$$

onde $(s=2,3),(j=$ PIP, DIP) e $(v=P, M)$, nessa ordem.

$$
{ }^{3}-1 T_{\beta}\left(\theta_{\beta, D}\right)=\left[\begin{array}{cccc}
1 & 0 & 0 & d_{\beta, D} \\
0 & 1 & 0 & 0 \\
0 & 0 & 1 & 0 \\
0 & 0 & 0 & 1
\end{array}\right]
$$

\subsubsection{Cinemática dos dedos anelar e mínimo}

Para os dedos anelar e mínimo, a cinemática direta é definida de acordo com os parâmetros da Tabela 4, lembrando que são 4 juntas e 4 links por dedo. A Equação (12) exibe as matrizes que contêm a posição e a orientação das pontas dos dedos com respeito ao centro do punho, onde $(\gamma=\mathrm{R}, \mathrm{L}),($ Ring, Little).

Tabela 4 Parâmetros D-H para os dedos anelar e mínimo

$$
\begin{array}{cccccc}
\hline \text { junta } & \theta_{\mathrm{i}, \mathrm{i}} & \mathrm{d}_{\mathrm{i}, \mathrm{i}} & \mathrm{r}_{\mathrm{i}, \mathrm{i}} & \alpha_{\mathrm{i}, \mathrm{i}} \\
\hline \mathrm{W} & 0 & 0 & \mathrm{r}_{\gamma, \mathrm{W}} & 0 \\
1 & \theta_{\gamma, C M C} & 0 & \mathrm{r}_{\gamma, M C} & \alpha_{\gamma, M C} \\
2 & \theta_{\gamma, M C P} & 0 & \mathrm{r}_{\gamma, \mathrm{P}} & \alpha_{\gamma, \mathrm{P}} \\
3 & \theta_{\gamma, \mathrm{PIP}} & \mathrm{d}_{\gamma, \mathrm{P}} & 0 & 0 \\
4 & \theta_{\gamma, \mathrm{DIP}} & \mathrm{d}_{\gamma, \mathrm{M}} & 0 & 0 \\
5 & \theta & \mathrm{d}_{\gamma, \mathrm{D}} & 0 & 0 \\
P_{\gamma}={ }_{0}^{\omega} T_{\gamma}\left(r_{\gamma, \omega}\right){ }_{1}^{0} T_{\gamma}\left(\theta_{\gamma, C M C}\right){ }_{2}^{1} T_{\gamma}\left(\theta_{\gamma, M C P}\right){ }_{3}^{2} T_{\gamma}\left(\theta_{\gamma, P I P}\right){ }_{4}^{3} T_{\gamma}\left(\theta_{\gamma, D I P}\right){ }_{5}^{4} T_{\gamma}\left(\theta_{\gamma, D I P}\right)
\end{array}
$$

Aqui, $\mathrm{P}_{\gamma}$ representa as matrizes que contêm a posição e a orientação das pontas dos dedos com respeito ao centro do punho; $\mathrm{T}_{\gamma}$ são as matrizes como aquelas da Equação (2), aqui dadas por: 


$$
\begin{gathered}
{ }_{0}^{\omega} T_{\gamma}\left(r_{\gamma, \omega}\right)=\left[\begin{array}{cccc}
1 & 0 & 0 & 0 \\
0 & 1 & 0 & 0 \\
0 & 0 & 1 & r_{\gamma, \omega} \\
0 & 0 & 0 & 1
\end{array}\right] \\
{ }_{s}^{s-1} T_{\gamma}\left(\theta_{\gamma, j}\right)=\left[\begin{array}{cccc}
\cos \left(\theta_{\gamma, j}\right) & -\sin \left(\theta_{\gamma}, j\right) & 0 & 0 \\
\cos \left(\alpha_{\gamma, v}\right) \sin \left(\theta \gamma_{, j}\right) & \cos \left(\alpha_{\gamma, v}\right) \cos \left(\theta_{\gamma, j}\right) & -\sin \left(\alpha_{\gamma, v}\right) & -r_{\gamma, v} \sin \left(\alpha_{\gamma, v}\right) \\
\sin \left(\alpha_{\gamma, v}\right) \sin \left(\theta \gamma_{, j}\right) & \sin \left(\alpha_{\gamma, v}\right) \cos \left(\theta_{\gamma, j}\right) & \cos \left(\alpha_{\gamma, v}\right) & r_{\gamma, v} \cos \left(\alpha_{\gamma, v}\right) \\
0 & 0 & 0 & 1
\end{array}\right]
\end{gathered}
$$

onde $(s=1,2),(v=M C, P)$ e $(j=C M C, M C P)$, nessa ordem.

$$
{ }_{b}^{b-1} T_{\gamma}\left(\theta_{\gamma, k}\right)=\left[\begin{array}{cccc}
\cos \left(\theta_{\gamma, k}\right) & -\sin \left(\theta_{\gamma, k}\right) & 0 & d_{\gamma, \omega} \\
\sin \left(\theta_{\gamma, k}\right) & \cos \left(\theta_{\gamma, k}\right) & 0 & 0 \\
0 & 0 & 1 & 0 \\
0 & 0 & 0 & 1
\end{array}\right]
$$

onde $(\mathrm{h}=1,2),(\mathrm{w}=\mathrm{P}, \mathrm{M})$ e $(\mathrm{k}=\mathrm{PIP}, \mathrm{DIP})$, nessa ordem.

$$
{ }_{5}^{4} T_{\gamma}\left(\theta_{\gamma, D}\right)=\left[\begin{array}{cccc}
1 & 0 & 0 & d_{\gamma, D} \\
0 & 1 & 0 & 0 \\
0 & 0 & 1 & 0 \\
0 & 0 & 0 & 1
\end{array}\right]
$$

\section{SIMULAC̣ÃO COMPUTACIONAL}

Com base no modelo matemático descrito anteriormente, são realizadas simulações computacionais para comparar o modelo real ao modelo matemático na realização de alguns movimentos de preensão da mão. Em posse do modelo matemático, foi possível implementar as equações do movimento e simular algumas situações de preensão da prótese. As medidas reais da prótese foram utilizadas como parâmetros do modelo, os quais são exibidos na Tabela 5. 
Tabela 5 Parâmetros da prótese

\begin{tabular}{|c|c|c|c|c|c|c|c|}
\hline \multicolumn{8}{|c|}{ Polegar } \\
\hline \multicolumn{2}{|c|}{$\theta_{i, i}$} & \multicolumn{2}{|c|}{$d_{i, j}$} & & \multicolumn{2}{|r|}{$\alpha_{i, i}$} \\
\hline$\theta_{T, T M C}$ & 0 a 40 & & & $r_{T, w}$ & $-304,5206$ & & \\
\hline$\theta_{\mathrm{T}, \mathrm{MCP}}$ & 0 a 60 & $d_{T, P}$ & 37 & $r_{T, M C}$ & $-383,8076$ & $\alpha_{T, M C}$ & 3,0466 \\
\hline$\theta_{\mathrm{T}, \mathrm{P}}$ & 0 a 60 & $d_{T, D}$ & 32 & $r_{T, P}$ & 42,4336 & $\alpha_{T, P}$ & 0,7321 \\
\hline \multicolumn{8}{|c|}{ Indicador } \\
\hline \multicolumn{2}{|c|}{$\theta_{i, j}$} & \multicolumn{2}{|c|}{$d_{i, i}$} & \multicolumn{2}{|r|}{$r_{i, i}$} & \multicolumn{2}{|r|}{$\alpha_{i, i}$} \\
\hline$\theta_{1, M C P}$ & 0 a 100 & $d_{1, P}$ & 38 & $r_{\mathrm{l}, \mathrm{w}}$ & 92 & \multirow{3}{*}{$\alpha_{1, M C}$} & \multirow{3}{*}{-1.6581} \\
\hline$\theta_{\mathrm{I}, \mathrm{PIP}}$ & 0 a 80 & $d_{l, M}$ & 24 & $r_{1, M C}$ & 40,1528 & & \\
\hline$\theta_{\mathrm{I}, \mathrm{DIP}}$ & 0 a 60 & $d_{1, D}$ & 30,5 & & & & \\
\hline \multicolumn{8}{|c|}{ Médio } \\
\hline \multicolumn{2}{|c|}{$\theta_{i, j}$} & \multicolumn{2}{|c|}{$d_{i, j}$} & \multicolumn{2}{|r|}{$r_{i, i}$} & \multicolumn{2}{|r|}{$\alpha_{i, i}$} \\
\hline$\theta_{M, M C P}$ & 0 a 100 & $d_{M, P}$ & 41 & \multirow{3}{*}{$\begin{array}{r}r_{M, w} \\
r_{M, M C}\end{array}$} & 96 & \multirow{3}{*}{$\alpha_{M, M C}$} & \multirow{3}{*}{-1.6406} \\
\hline$\theta_{M, P I P}$ & 0 a 80 & $d_{M, M}$ & 27 & & 13,0317 & & \\
\hline$\theta_{M, \mathrm{DIP}}$ & 0 a 60 & $d_{M, D}$ & 34 & & & & \\
\hline \multicolumn{8}{|c|}{ Anelar } \\
\hline \multicolumn{2}{|c|}{$\theta_{i, i}$} & \multicolumn{2}{|c|}{$d_{i, j}$} & \multicolumn{2}{|r|}{$r_{i, i}$} & \multicolumn{2}{|r|}{$\alpha_{i, i}$} \\
\hline$\theta_{R, C M C}$ & 0 a 25 & & & $r_{R, w}$ & 52,8391 & & \\
\hline$\theta_{R, M C P}$ & 0 a 80 & $d_{R, P}$ & 33 & $r_{R, M C}$ & 65,1065 & $\alpha_{R, M C}$ & $-0,8727$ \\
\hline$\theta_{R, P I P}$ & 0 a 80 & $d_{R, M}$ & 22 & $r_{R, P}$ & $-65,4793$ & $\alpha_{R, P}$ & $-0,6028$ \\
\hline$\theta_{\mathrm{R}, \mathrm{DIP}}$ & 0 a 70 & $d_{R, D}$ & 28 & & & & \\
\hline \multicolumn{8}{|c|}{ Mínimo } \\
\hline \multicolumn{2}{|c|}{$\theta_{i, j}$} & \multicolumn{2}{|c|}{$d_{i, j}$} & \multicolumn{2}{|r|}{$r_{i, i}$} & \multicolumn{2}{|r|}{$\alpha_{i, i}$} \\
\hline$\theta_{L, C M C}$ & 0 a 25 & & & $r_{L, w}$ & 55,4641 & & \\
\hline$\theta_{L, M C P}$ & 0 a 80 & $d_{L, P}$ & 31 & $r_{L, M C}$ & 49,0825 & $\alpha_{L, M C}$ & $-0,6778$ \\
\hline$\theta_{\mathrm{L}, \mathrm{PIP}}$ & 0 a 80 & $d_{L, M}$ & 19 & $r_{L, P}$ & $-74,5525$ & $\alpha_{L, P}$ & $-0,6480$ \\
\hline$\theta_{L, D I P}$ & 0 a 70 & $d_{L, D}$ & 25 & & & & \\
\hline
\end{tabular}

A construção do protótipo será detalhada na Seção 4. As medidas foram tomadas em milímetros e os ângulos em graus. A origem do sistema de coordenadas é posicionada no dentro da linha da base do punho, em que o plano $x y$ é considerado como sendo o plano médio da prótese. 
Sob a taxonomia proposta por Cutkosky (1989), os apertos da mão são divididos em apertos de força e apertos de precisão. Nos apertos de precisão, a sensibilidade e a destreza são predominantes, enquanto nos apertos de força têm-se grandes áreas de contato entre o objeto segurado e as superfícies dos dedos e da palma da mão. Nesse último grupo, pouca ou nenhuma habilidade é necessária para executar os movimentos.

$\mathrm{Na}$ Figura 5, é apresentada a prótese sem qualquer flexão nas juntas $\left(\theta_{\mathrm{ij}}=0\right.$ para todos i, j) e uma simulação desta situação no modelo implementado.

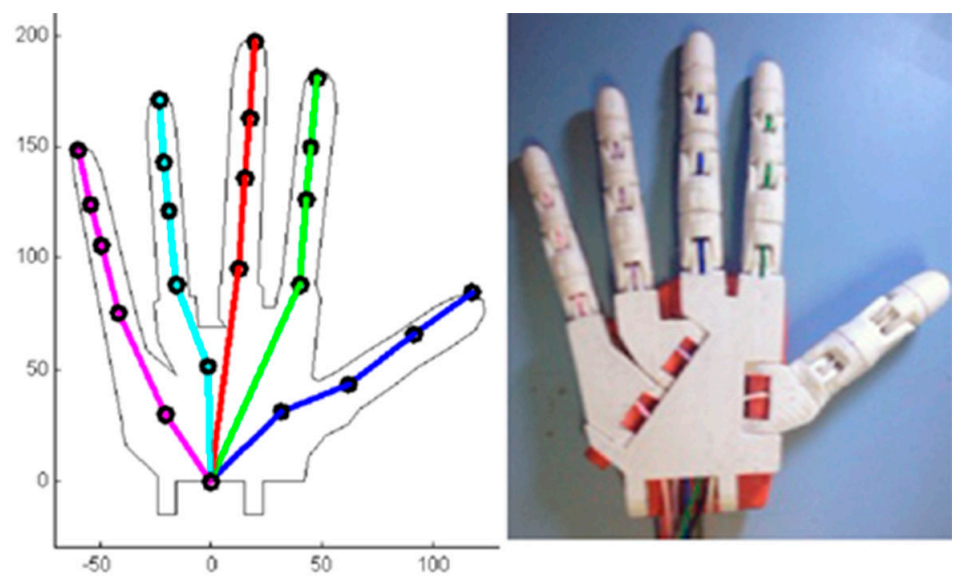

Figura 5 Modelo simulado e prótese em qualquer flexão dos dedos.

Na Figura 6, é apresentada uma situação na qual o polegar e o indicador estão em flexão total de todas suas juntas. Pode-se perceber que existe uma dificuldade geométrica para executar apertos de precisão. Aqui não foi considerada a análise de contato entre os dedos.

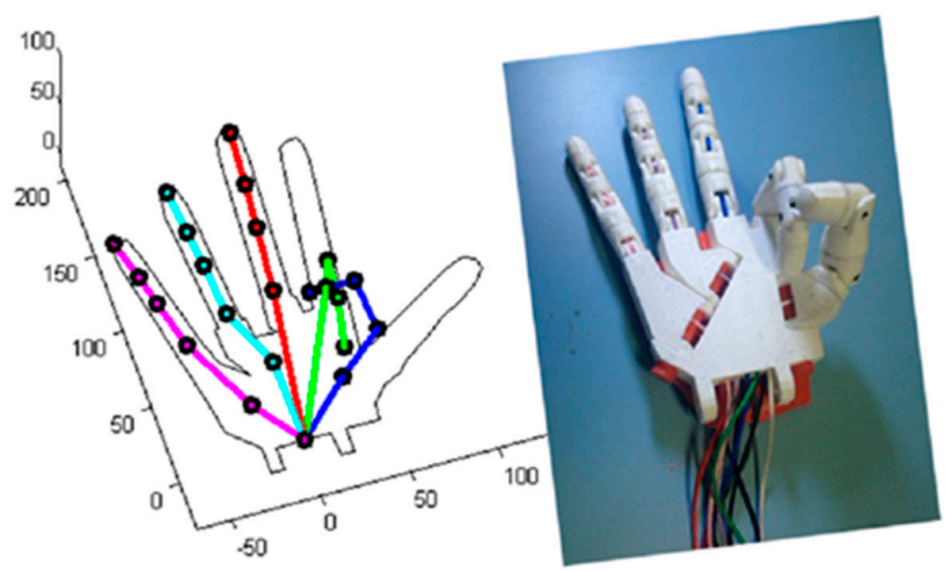

Figura 6 Modelo simulado e prótese com o polegar e indicador completamente flexionados. 
$\mathrm{Na}$ Figura 7, tem-se a prótese completamente flexionada (ângulos máximos em todas as juntas) e sua correspondente situação modelada.

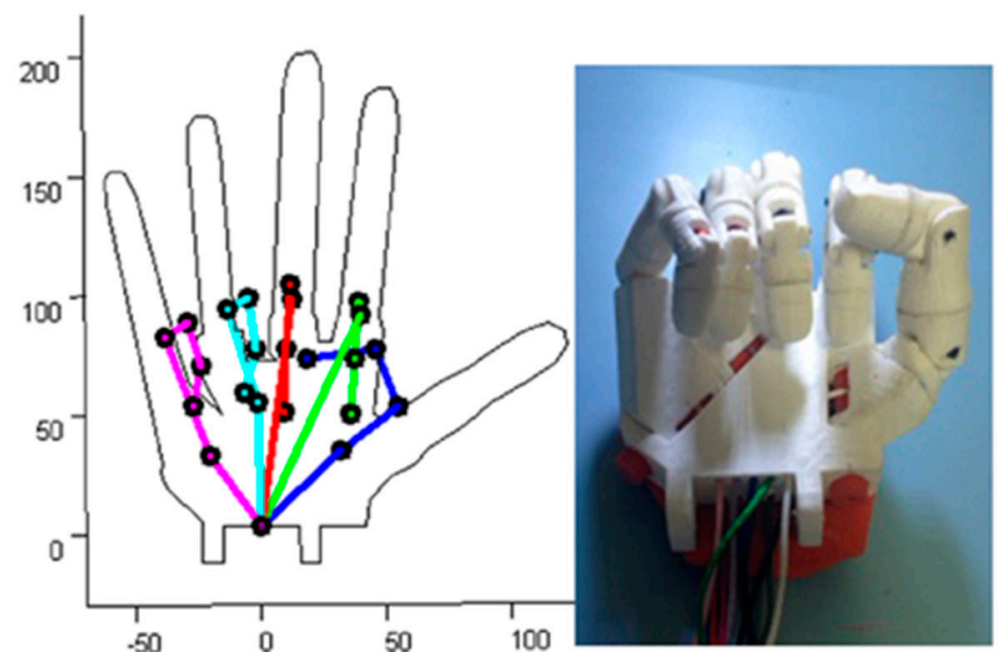

Figura 7 Modelo simulado e prótese completamente flexionada.

\section{CONSTRUC̣ÃO DO PROTÓTIPO}

De acordo com Smagt et al. (2009), existem algumas próteses de mão comerciais, tais como Vincent (Vincent Systems), iLimb e iLimb Pulse (Touch Bionics), Bebionic (RSL Steeper) e Michelangelo (Otto Bock). Contudo, o maior impedimento é que as próteses e braços robóticos são muito caros (ALLIN et al., 2010).

Dentre os vários trabalhos na área de próteses de mão, existem aqueles com foco na análise mecânica, outros apenas no projeto cinemático. Smagt et al. (2009) apontam que, mais do que copiar a estrutura intrínseca da mão, no projeto da prótese, o objetivo é copiar o mais fielmente possível as propriedades da mão humana real. Du e Charbon (2007) apresentam um modelo de ajuste 3D de mão que pode reproduzir as posições dos dedos com precisão. Gosselin et al. (2008) apresentam uma geometria ótima de dedos movidos por tendões para um protótipo de prótese de mão antropomórfica com 15 GDL e um único atuador. Dalley et al. (2009) desenvolvem uma prótese de mão humana com 16 juntas movidas por um conjunto de 5 atuadores independentes, em que cada junta dos dedos possui uma mola torsional. Gaiser et al. (2009) fazem uma combinação de atuadores fluídicos flexíveis e elementos passivos leves para reduzir a força necessária para a pegada de diferentes objetos. Dechev et al. (2001) apresentam uma prótese de mão experimental, com múltiplos dedos, projetada para um grupo de crianças entre 7 e 11 anos de idade, que resultou em uma mão com peso e tama- 
nho reduzido, apropriada para crianças. Baril et al. (2013) propõem um pegador antropomórfico para aplicações prostéticas, cujo acionador mecânico é montado na palma da mão, de modo a permitir uma distribuição apropriada das forças com consequente vantagem mecânica.

Com respeito à forma, de acordo com Belter et al. (2013), o projeto da prótese deve levar em conta o peso do sistema inteiro, além da fonte de energia (bateria) que deve ser carregada pelo usuário. Dessa forma, o peso é uma das características mais importantes nesse desenvolvimento. Anatomicamente, existe mais ou menos independência entre os dedos da mão. Nesse sentido, Ingram et al. (2008) afirmam que o polegar é o dedo mais importante, enquanto o anelar é o menos importante, dentre todos os dedos da mão. O polegar carrega cerca de $40 \%$ de toda a funcionalidade da mão humana. Portanto, é claro que o posicionamento do polegar deve ser minuciosamente avaliado, de modo a permitir um número considerável de possibilidades de apertos e manuseio de objetos.

Na sequência, são exibidos os procedimentos construtivos da mão avaliada, cujos componentes estruturais são manufaturados em impressora 3D, seguidos do detalhamento do sistema eletrônico de controle. Conforme mencionado anteriormente, foi utilizado o projeto InMoov, de código aberto. Os arquivos (STL) com as partes integrantes do braço e mão robóticos foram obtidos no site do projeto (LANGEVIN, 2014). Optou-se por construir uma mão direita, de que a Figura 8 exibe o conjunto das peças que foram impressas.

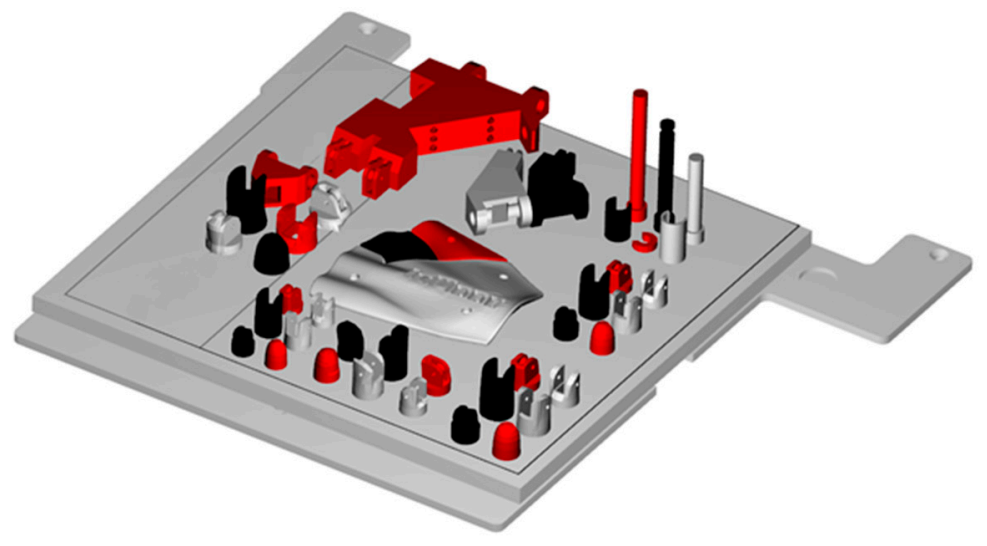

Figura 8 Conjunto de peças impressas para a composição da mão.

Na sequência, as partes foram impressas em impressora 3D, modelo CubeX, que possui 3 cabeças de impressão, fabricada pela 3DSystems, utilizando material plástico biodegradável PLA. Foram utilizadas as cores branca, preta, vermelha e natural para a impressão das peças, no que foi observado que a cor influencia a 
resistência mecânica e o acabamento das peças. Diversos ajustes de montagem, para o perfeito encaixe das peças precisaram ser executados. A Figura 9 exibe o polegar e o indicador montados. Para a união das peças, foi utilizada cola Super Bonder $^{\circledR}$ e nas juntas articuladas foram utilizados pedaços de filamentos de material PLA, o mesmo utilizado na impressão de todas as outras partes. $\mathrm{O}$ acabamento externo desses filamentos foi realizado com ferro de solda comumente utilizado em eletrônica. A Figura 10 exibe a mão, em que, nos dedos indicador e médio, é possível observar os filamentos nas juntas ainda sem o acabamento externo.

Inicialmente foi utilizado fio de nylon para realizar a atuação dos dedos, em movimento de flexão e extensão (Figura 9). Na montagem da mão completa, foram utilizados fios de seda coloridos, responsáveis pela flexão e extensão dos dedos, de modo a facilitar a identificação da ação de cada um dos dedos (Figura 10).

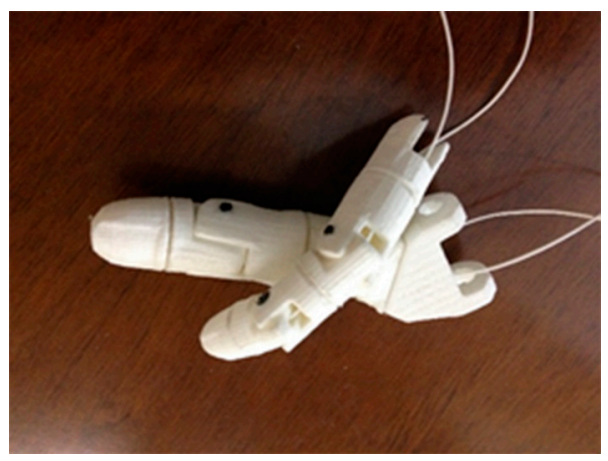

Figura 9 Polegar e indicador impressos em PLA branco (fios de nylon branco para movimento).

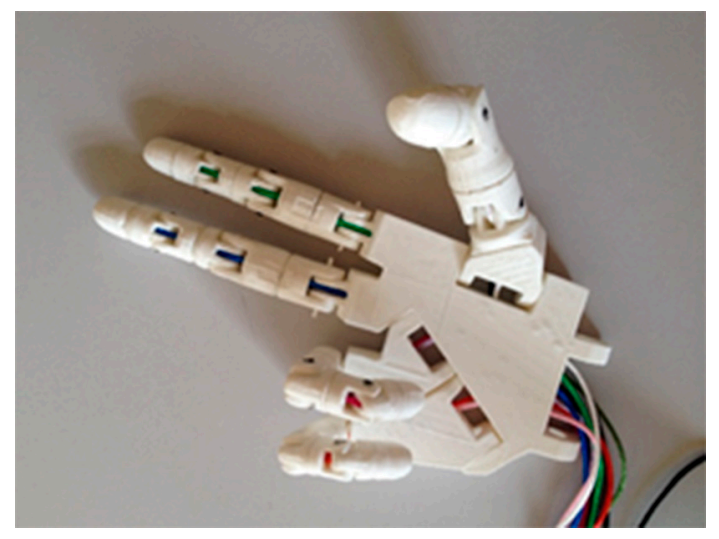

Figura 10 Mão completa impressa em PLA branco (fios de seda coloridos para movimento).

Para finalizar o protótipo, foi construído o sistema de suporte e acoplamento dos servomotores que fazem o movimento dos dedos. Para tanto, foram impres- 
sas as peças que compõem o antebraço e o punho que sustentam a mão, além do sistema de acoplamento para servos e cabos.

O punho é dotado de um servomotor extra, que, por meio de um sistema de engrenagens, permite o movimento rotacional da mão em torno do eixo longitudinal do antebraço.

Foram utilizados servomotores da marca TowerPro, modelo MG946, dotado de engrenagens de metal, $55 \mathrm{~g}$, alto torque, $12 \mathrm{~kg} . \mathrm{cm}$, velocidade de operação de $0,20 \mathrm{seg} / 60$ graus. O movimento de cada dedo é realizado por um servomotor, por meio de 2 cabos para flexão e extensão, respectivamente. Portanto, para o movimento dos dedos a prótese tem 5 servomotores alocados no antebraço, e um outro servomotor no punho para rotação da mão. Os fios de seda foram substituídos por cabos de aço com revestimento plástico externo, conforme pode ser observado na Figura 11.

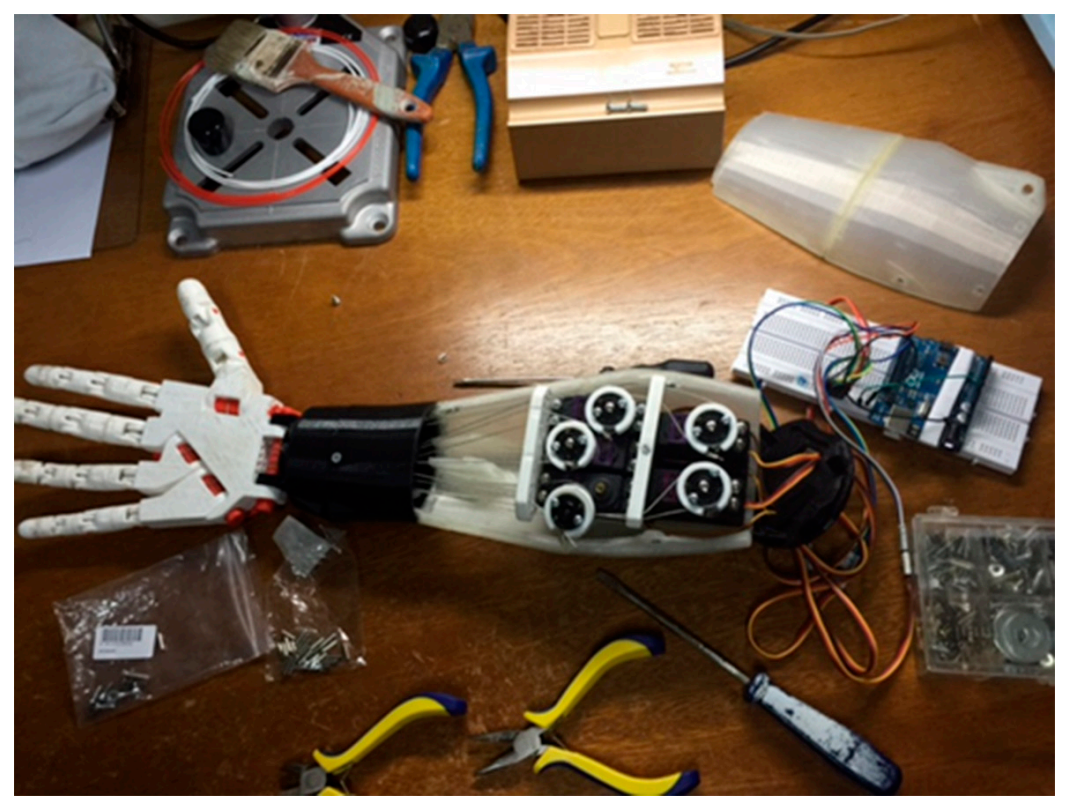

Figura 11 Prótese com vista interna dos servomotores alocados no antebraço.

\section{SISTEMA DE CONTROLE}

Para controle do movimento dos dedos, foi utilizada uma placa Arduino UNO Rev3. O primeiro teste de controle foi realizado ligando-se diretamente os servos ao Arduino, conforme diagrama esquemático apresentado na Figura 12. A conexão física deu-se por meio de uma protoboard, cujo esquema de ligação dos fios é exibido na Figura 13. 


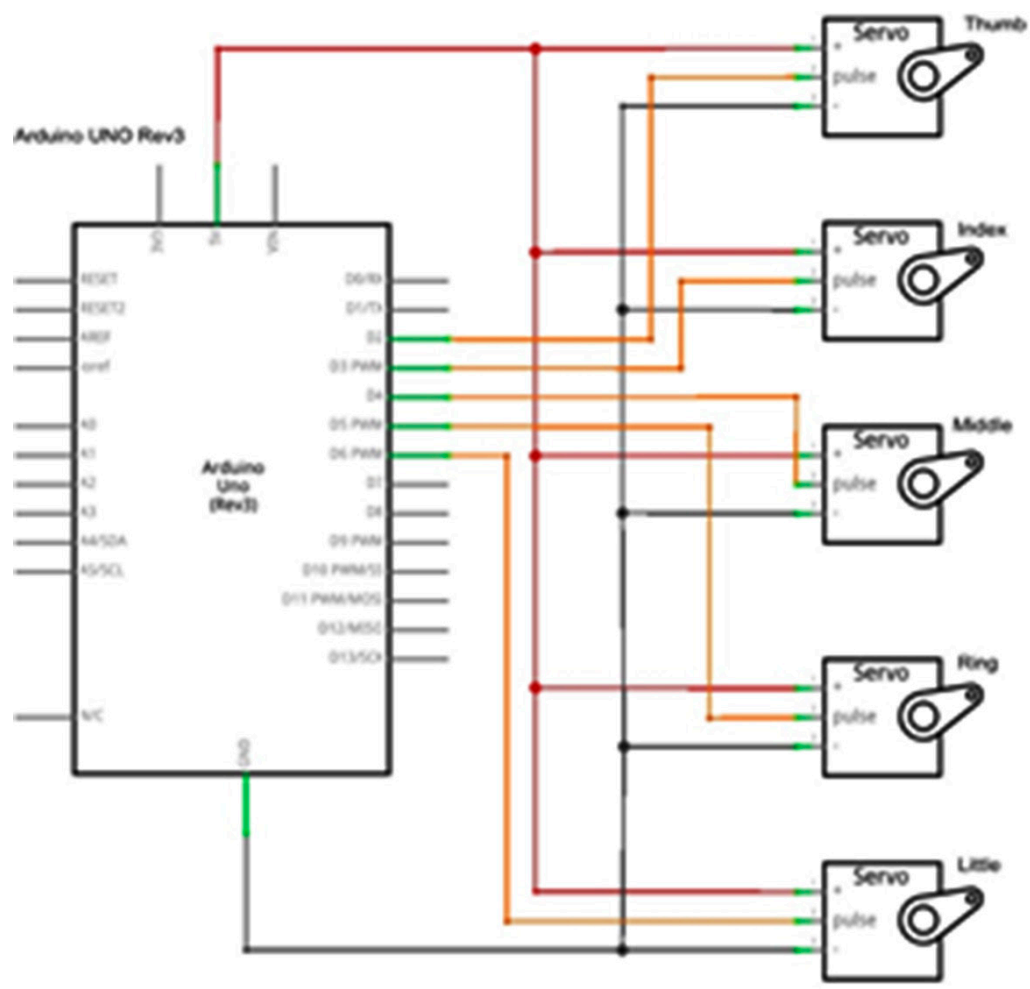

Figura 12 Diagrama esquemático - teste 1.

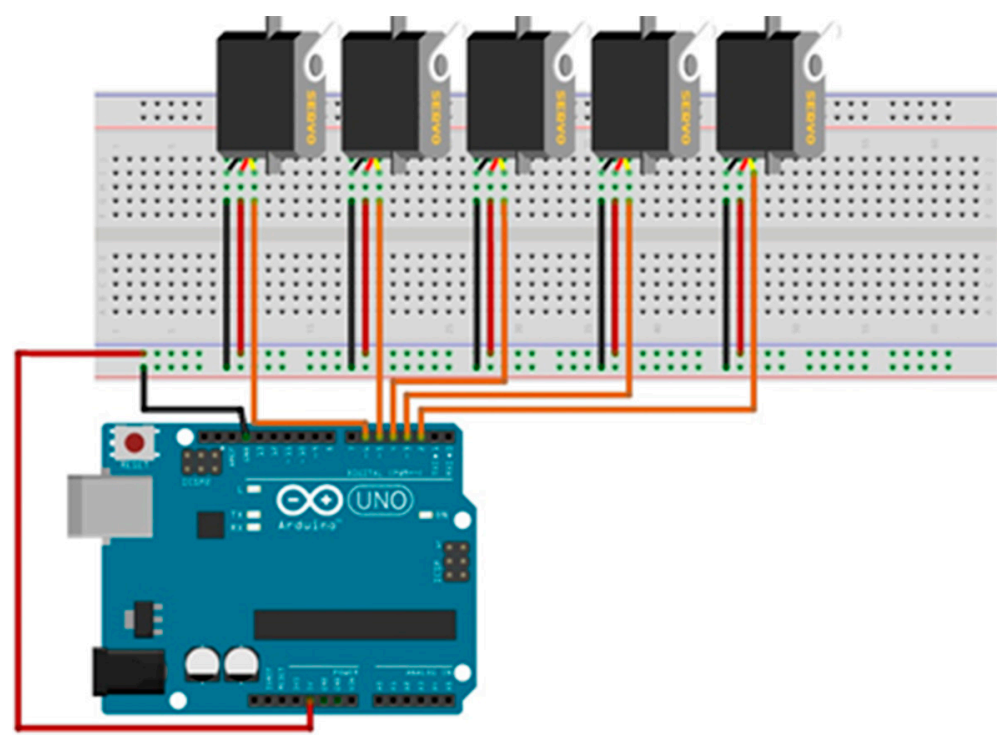

Figura 13 Esquema de conexão entre Arduino, protoboard e servos. 
A programação para esse teste realiza (em loop) movimento completo de flexão e extensão de cada um dos dedos, na sequência: polegar, indicador, médio, anelar e mínimo. Convém mencionar que, nesse caso, apenas um servo é acionado por vez.

Com o intuito de obter um maior controle da prótese, foi realizado um novo teste, em que o controle dos dedos é dado pelo teclado do computador. O sistema de controle é realizado por meio das teclas conforme Tabela 6 .

Tabela 6 Indicação de teclas para controle dos dedos

\begin{tabular}{cccccc} 
& polegar & indicador & médio & anelar & mínimo \\
\hline flexão & $\mathrm{A}$ & $\mathrm{S}$ & $\mathrm{D}$ & $\mathrm{F}$ & $\mathrm{G}$ \\
extensão & $\mathrm{Q}$ & $\mathrm{W}$ & $\mathrm{E}$ & $\mathrm{R}$ & $\mathrm{T}$ \\
\hline
\end{tabular}

Contudo, constatou-se que a potência elétrica fornecida pelo Arduino não era suficiente para movimentar 3 ou mais servos simultaneamente. Então, o circuito foi modificado e foi adicionada uma nova fonte de energia, no caso, uma bateria $6.0 \mathrm{~V}, 2200 \mathrm{mAh}$, ligada a um regulador de tensão. Assim, apenas o sistema de controle de pulso dos servos é conectado ao Arduino, conforme o diagrama esquemático da Figura 14.

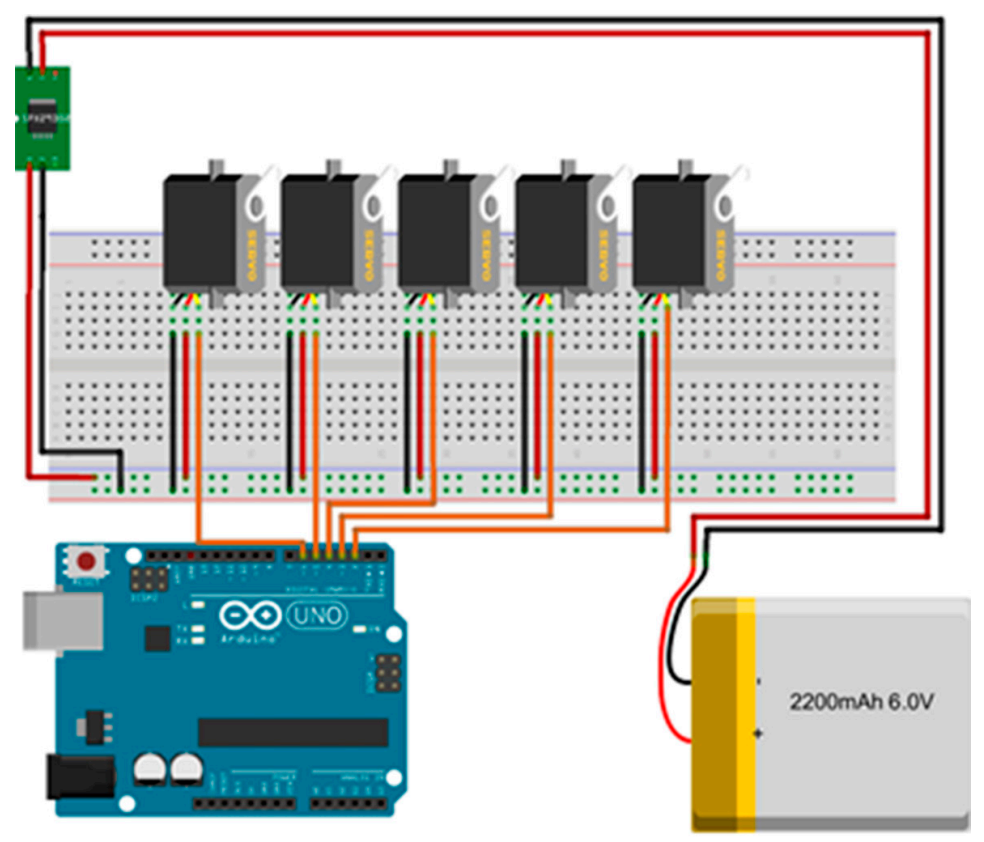

Figura 14 Diagrama esquemático do teste 2. 


\section{CONCLUSÕES}

De acordo com Belter et al. (2013), a relação entre o eixo de rotação do polegar e o eixo principal do punho é um parâmetro crítico e é fundamental para a trajetória do polegar, e consequentemente para o desempenho da maioria dos movimentos de preensão e manipulação de objetos. Na prótese de mão construída e analisada aqui, o eixo de rotação do polegar é coincidente com o eixo longitudinal do dedo indicador. Esse é um problema na reprodução de alguns tipos de pegadas. Um grande número de movimentos da mão envolve o polegar e o indicador. De modo simplificado, os eixos longitudinais dos dedos apontam para o centro do punho.

Neste trabalho, foi analisada e construída uma mão robótica desenvolvida pelo projeto Opens Source InMoov (LANGEVIN, 2014), composta por 5 dedos independentes, num total de 17 GDL. Foi desenvolvido um modelo cinemático completo para a mão robótica, cujas equações foram determinadas pelas convenções de Denavit-Hartenberg.

No caso da simulação computacional realizada, existem algumas diferenças consideráveis entre os modelos real e virtual, devido a simplificações e características que não foram consideradas na simulação. Contudo, com o modelo computacional foi possível simular alguns tipos comuns de movimentos da mão, comparando os dois modelos.

A construção, a utilização e os testes com a mão robótica InMoov apontaram que ela não pode ser usada diretamente como uma prótese antropomórfica. Assim, vários ajustes precisam ser efetuados para aumentar a mobilidade e a facilidade de uso. Outro fator que é um impeditivo para o uso desta como prótese é o peso, que se mostra excepcionalmente alto. Sabe-se que um dos principais fatores que fazem com que os usuários abandonem o uso das próteses é o peso que estas possuem.

Com a experiência adquirida devido aos testes efetuados neste projeto, novas investigações são naturalmente apontadas, como alterações na geometria da prótese de modo que os eixos longitudinais dos dedos apontem para o centro do punho e os eixos dos dedos indicador e médio não sejam paralelos. Além disso, é necessária a diminuição do peso total da prótese, alocando os motores na palma da mão, com acionamento dos dedos por meio de engrenagens, por exemplo. Um outro sistema a ser estudado é a inclusão de sensores de força nas pontas dos dedos, que poderá fornecer um melhor parâmetro de controle de acordo com os objetos em preensão. No contexto do controle, está sendo desenvolvido controle por tecnologia embarcada (celular e tablete), por meio de comandos de voz que servirão de apoio à utilização de myosensores, para acoplamento da prótese com os músculos do antebraço. 


\section{AGRADECIMENTOS}

Os autores agradecem o suporte financeiro da CAPES por meio de bolsa do programa PNPD e do CNPq por meio do edital MCTI-SECIS-CNPq-84-2013, proc. 458649/2013-9, e ainda o apoio recebido da UFU, UFG/RC, FEMEC, FAPEG e FAPEMIG.

\section{REFERÊNCIAS}

ALLIN, S. et al. Recent trends in the development an devaluation of assistive robotic manipulation devices. Physical Medicine and Rehabilitation Clinics of North America, $\mathrm{n}$. 50, p. 59-77, 2010.

BARIL, M. et al. On the design of a mechanically programmable underactuated anthropomorphic prosthetic gripper, v. 135, 2013.

BELTER, J. et al. Mechanical design and performance specifications of anthropomorphic prosthetic hands: A review. JRRD-Journal of Rehabilitation Research \& Development, $\mathrm{n}$. 50 v. 5, p. 599-618, 2013.

CARVALHO, J. Amputações de membros inferiores: em busca da plena reabilitação. Barueri: Manole, 2003.

COBOS, S. et al. Efficient human hand kinematics for manipulation tasks. IEEE/RSJ International Conference on Intelligent Robots and Systems, Nice, 2008.

. Simplified Human Hand Models for Manipulation Tasks, Cutting Edge Robotics 201, p. 155-174, 2010.

CRAIG, J. Introduction to robotics: mechanics and control. 3. ed. New York: Prentice Hall, 2004.

CUTKOSKY, M. R.; WRIGHT, P. K. Modeling manufacturing grips and correlations with design of robotic hands. IEEE International Conference on Robotics and Automation, p. 1533-1539, 1986.

CUTKOSKY, M. R. On grasp choice, grasp models, and the design of hands for manufacturing tasks. IEEE Transactions on Robotics and Automation, n. 5, v. 3, p. 269278, 1989.

DALLEY, S. et al. Design of a multifunctional anthropomorphic prosthetic hand with extrinsic actuation. IEEE/ASME Transactions on Mechatronics, n. 14, v. 6, p. 699-706, 2009.

DECHEV, N.; CLEGHORN, W.L.; NAUMANN, S. Multiple finger, passive adaptive grasp prosthetic hand. Mechanism and Machine Theory, n. 36, p. 1157-1173, 2001.

DELLON, B.; MATSUOKA, Y. Prosthetics, exoskeletons, and reahabilitation: now and for the Future. IEEE Robotics \& Automations Magazine, p. 30-34, 2007.

DU, H.; CHARBON, E. 3d hand model fitting for virtual keyboard system. IEEE Workshop on Applications of Computer Vision (WACV'07), 2007.

GAISER, I. et al. The fluid hand iii: a multifunctional prosthetic hand. Journal of Prosthetics and Orthics, n. 21, v. 2, p. 1-6, 2009. 
GREBENSTEIN, M. et al. A Method for Hand Kinematics Designers 7 Billion Perfect Hands, ICABB-2010, Venice, 2010.

GOSSELIN, C.; PELLETIER, F.; LALIBERTÉ, T. An anthropomorphic under actuated robotic hand with 15 dofs and a single actuator. 2008.

HARVEY, D.; LONGSTAFF, B. The Development of a Prosthetic Arm, Department of Mechanical Engineering, The University of Adelaide, Adelaide.

Disponível em: http://citeseerx.ist.psu.edu/viewdoc/download?doi=10.1.1.132.9852\&rep =rep1\&type=pdf. Acesso em: 04 Oct. 2013.

INGRAM, J. et al. The statistics of natural hand movements. Experimental Brain Research, n. 188, p. 223-236, 2008.

KHALIL, W.; KLEINFINGER, J. A new geometric rotation for open closed-loop robots. IEEE International Conference on Robotics and Automation, v. 1, n. 3, p. 1174-1179, 1986.

KULLEY, M. Hand Prosthetics - Statistics. 2003.

Disponível em:

$<$ http://biomed.brown.edu/Courses/BI108/BI108_2003_Groups/Hand_Prosthetics/stats. html>. Acesso em: 04 Oct. 2013.

LANGEVIN, G. Projeto opens source InMoov. 2014. Disponível em: <http://www. inmoov.fr/project/>. Acesso em: 26 jul. 2016.

O'SULLIVAN, S.; SCHMITZ, T. Fisioterapia: avaliação e tratamento. 4. ed. Barueri: Manole, 2004.

PELEG, D. et al. Classification of Finger Activation for Use in a Robotic Prosthesis Arm. IEEE Transactions on Neural Systems and Rehabilitation Engineering, v. 10, n. 4, 2002.

SHENOY, P. et al. Online Electromyographic Control of a Robotic Prosthesis. IEEE Transactions on Biomedical Engineering, v. 55, n. 3, 2008.

SHIBATA, L.; MENDES, V. L. F. (Org.). Diretrizes de Atenção à Pessoa Amputada. Ministério da Saúde. Secretaria de Atenção à Saúde. Departamento de Ações Programáticas Estratégicas. Brasília, DF: Ministério da Saúde, 2012.

SMAGT, P. et al. Robotics of human movements. Journal of Physiology, New York, n. 103, p. 119-132, 2009.

TARZIMI, W.; ELAMVAZUTHI, I.; BEGAM, M. Kinematic and dynamic modeling of a multi-fingered robot hand. International Journal of Basics \& Applied Sciences, New York, v. 9, p. 61-68, 2009.

VENTIMIGLIA, P. Design of a Human Hand Prosthesis. Major Qualifying Project Report. Faculty of the Worcester Polytechnic Institute, Worcester, 2012.

WEIR, R. F. F. F. Design of artificial arms and hands for prosthetic applications. Standard Handbook of Biomedical Engineering and Design. New York: McGraw-Hill, 2004. 
